\title{
Dopa-responsive dystonia due to sepiapterin reductase deficiency
}

INSERM

\section{Source}

INSERM. (1999). Orphanet: an online rare disease and orphan drug data base. Doparesponsive dystonia due to sepiapterin reductase deficiency. ORPHA:70594

Dopa-responsive dystonia (DRD) due to sepiapterin reductase deficiency (SRD) is a very rare neurometabolic disorder characterized by dystonia with diurnal fluctuations, axial hypotonia, oculogyric crises, and delays in motor and cog nitive development. 